\title{
Solving fuzzy fractional differential equations using fuzzy Sumudu transform
}

\author{
Norazrizal Aswad Abdul Rahman, Muhammad Zaini Ahmad* \\ Institute of Engineering Mathematics, Universiti Malaysia Perlis, Pauh Putra Main Campus, 02600 Arau, Perlis, Malaysia. \\ Communicated by W. Shatanawi
}

\begin{abstract}
In this paper, we apply fuzzy Sumudu transform (FST) for solving linear fuzzy fractional differential equations (FFDEs) involving Caputo fuzzy fractional derivative. It is followed by suggesting a new result on the property of FST for Caputo fuzzy fractional derivative. We then construct a detailed procedure on finding the solutions of linear FFDEs and finally, we demonstrate a numerical example. (C)2017 All rights reserved.
\end{abstract}

Keywords: Caputo fuzzy fractional derivative, fuzzy Sumudu transform, fuzzy fractional differential equation. 2010 MSC: 26A33, 44A05, 34A08, 34A07.

\section{Introduction}

Fractional calculus is the generalization of ordinary calculus. This includes the functions' derivative of arbitrary order. The topic has been explored and studied by various researchers in many fields such as engineering, mathematics and so forth $[2,14,15,17,22,33,35]$. One of the major contributions in this field was the work in [37], which discussed the topic intensively. Later, it was studied in [31], where the authors proposed some applications. When dealing with fractional differential equations, the terms such as Riemann-Liouville, Grünwald-Letnikov and Caputo fractional derivative are considered by many authors [21, 25, 30, 39, 42]. Of the three definitions for derivative stated, Riemann-Liouville and Caputo fractional derivatives appeared to be more popular.

As times moving on, the fractional differential equations seem to have some drawbacks. One of them is the initial value assigned to the model. In general, the determination of initial values is very difficult. It always involves uncertainty quantities. This is true when dealing with real physical phenomena. To handle uncertainty quantities, researchers proposed several new concepts. The one that stands out among the concepts is fuzzy set theory [48]. This theory is able to deal with differential equations possessing uncertainties at initial values. The first contribution on handling fractional differential equations with

\footnotetext{
*Corresponding author

Email addresses: norazrizalaswad@gmail.com (Norazrizal Aswad Abdul Rahman), mzaini@unimap.edu.my (Muhammad Zaini Ahmad)
}

doi:10.22436/jnsa.010.05.28 
uncertainties was studied in [3]. This has influenced many researchers to further explore the subject $[5,6,8,9,29,43]$.

Integral transforms have long been used in solving linear ordinary differential equations, as well as linear fractional differential equations. The integral transforms were preceded by Fourier transform. Later, several new integral transforms have been proposed, namely, Laplace, Mellin, and Hankel transforms $[32,40,44]$. One of the recent integral transforms introduced in the literature is the Sumudu transform [45]. The virtue of this transform is that it holds a scale preserving property which results in the original function to be similar with the transformed function. It can also be seen in the literature, there exist several discussions on solving few types of linear fractional differential equations, as we stated previously, using Sumudu transform [16, 18, 27]. Recently, fuzzy Laplace transform [7] has been used to solve FFDEs involving Riemann-Liouville fractional derivative [38]. However, this type of fractional derivative has a drawback. It requires a quantity of fractional H-derivative of an unknown solution at the fuzzy initial point, which is not practical in real life situation. In this paper, we propose a new solution of linear FFDEs involving Caputo fuzzy fractional derivative using FST. The FST is first proposed in [4] followed by [1]. Some other applications of FST can be seen in [23].

The arrangement of this paper is as the following. In Section 2, we revise some fundamental theories on fuzzy numbers and fuzzy functions. Plus, some definitions and theorems on Caputo fuzzy fractional derivative will also be provided. It is followed by the definition of FST in Section 3. In this section, we also propose a new property of FST for Caputo fuzzy fractional derivative. Next, in Section 4, we provide a procedure on solving linear FFDEs possessing Caputo fuzzy fractional derivative using the FST in detail. A numerical example is demonstrated in Section 5 and finally in Section 6, the conclusion is drawn.

\section{Basic concepts and theories}

Here, we revisit several definitions and theorems for a better understanding of this paper.

\subsection{Fuzzy numbers and fuzzy functions}

Throughout this paper, $\mathbb{R}$ denotes the set of real numbers. Fuzzy number is defined as follows.

Definition 2.1 ([47]). A fuzzy number is a mapping $\widetilde{u}: \mathbb{R} \rightarrow[0,1]$ with the following criteria.

1. $\tilde{u}$ is normal, i.e., there exists $x_{0} \in \mathbb{R}$ such that $\tilde{u}\left(x_{0}\right)=1$;

2. $\widetilde{u}$ is convex, i.e., for all and $\lambda \in[0,1], x, y \in \mathbb{R}$,

$$
\widetilde{u}(\lambda x+(1-\lambda) y) \geqslant \min \{\widetilde{u}(x), \widetilde{u}(y)\},
$$

holds;

3. $\widetilde{u}$ is upper semicontinuous, i.e., for any $x_{0} \in \mathbb{R}$,

$$
\widetilde{\mathfrak{u}}\left(x_{0}\right) \geqslant \lim _{x \rightarrow x_{0}^{ \pm}} \widetilde{\mathfrak{u}}(x) ;
$$

4. supp $\widetilde{\mathfrak{u}}=\{x \in \mathbb{R} \mid \widetilde{\mathfrak{u}}(x)>0\}$ is the support of $\widetilde{\mathfrak{u}}$, and its closure cl(supp $\widetilde{\mathfrak{u}})$ is compact.

Definition 2.2 ([26]). Let $\widetilde{u}$ be a fuzzy number defined in $\mathcal{F}(\mathbb{R})$. The $\alpha$-level set of $\widetilde{u}$, for any $\alpha \in[0,1]$, denoted by $\widetilde{u}_{\alpha}$, is a crisp set that contains all elements in $\mathbb{R}$, such that the membership value of $\widetilde{u}$ is greater or equal to $\alpha$, that is

$$
\widetilde{\mathfrak{u}}_{\alpha}=\{x \in \mathbb{R} \mid \widetilde{u}(x) \geqslant \alpha\} .
$$

Whenever we represent the fuzzy number with $\alpha$-level set, we can see that it is closed and bounded. It is denoted by $\left[\underline{u}_{\alpha}, \bar{u}_{\alpha}\right]$, where they represent the lower and upper bound $\alpha$-level set of a fuzzy number, respectively.

As the fuzzy number is resolved by the interval $\widetilde{u}_{\alpha}$, researchers [20,34] defined another representation, parametrically, of fuzzy numbers as in the following definition. 
Definition 2.3. A fuzzy number $\widetilde{u}$ in parametric form is a pair $\left[\underline{u}_{\alpha}, \bar{u}_{\alpha}\right]$ of functions $\underline{u}_{\alpha}$ and $\bar{u}_{\alpha}$ for any $\alpha \in[0,1]$, which satisfies the following requirements.

1. $\underline{\underline{u}}_{\alpha}$ is a bounded non-decreasing left continuous function in $(0,1]$;

2. $\bar{u}_{\alpha}$ is a bounded non-increasing left continuous function in $(0,1]$;

3. $\underline{u}_{\alpha} \leqslant \bar{u}_{\alpha}$.

Some researchers classified the fuzzy numbers into several types of fuzzy membership function. To the deepest of our study, triangular fuzzy membership function or also often referred to as triangular fuzzy number is the most widely used membership function.

Definition 2.4 ([28]). A triangular fuzzy number $\widetilde{u}$ can be defined by a triplet $\left(a_{1}, a_{2}, a_{3}\right)$, the membership function is defined as follows.

$$
\widetilde{u}(x)= \begin{cases}0, & \text { if } x<a_{1}, \\ \frac{x-a_{1}}{a_{2}-a_{1}}, & \text { if } a_{1} \leqslant x<a_{2}, \\ \frac{a_{3}-x}{a_{3}-a_{2}}, & \text { if } a_{2} \leqslant x \leqslant a_{3}, \\ 0, & \text { if } x>a_{3} .\end{cases}
$$

The $\alpha$-level of the fuzzy number $\widetilde{u}$ is $\widetilde{u}_{\alpha}=\left[a_{1}+\left(a_{2}-a_{3}\right) \alpha, a_{3}-\left(a_{3}-a_{2}\right) \alpha\right]$ for any $\alpha \in[0,1]$.

The definition of the operations on fuzzy numbers can be referred in [41].

Theorem 2.5 ([46]). Let $\tilde{\mathrm{f}}: \mathbb{R} \rightarrow \mathcal{F}(\mathbb{R})$ and it is represented by $\left[\underline{f}_{\alpha}(x), \overline{\mathrm{f}}_{\alpha}(\mathrm{x})\right]$. For any fixed $\alpha \in[0,1]$, assume $\underline{f}_{\alpha}(x)$ and $\bar{f}_{\alpha}(x)$ are Riemann-integrable on $[a, b]$ for every $b \geqslant a$, and assume there are two positive $\underline{M}_{\alpha}$ and $\bar{M}_{\alpha}$ such that $\int_{a}^{b}\left|\underline{f}_{\alpha}(x)\right| d x \leqslant \underline{M}_{\alpha}$ and $\int_{a}^{b}\left|\bar{f}_{\alpha}(x)\right| d x \leqslant \bar{M}_{\alpha}$ for every $b \geqslant a$. Then, $\widetilde{f}(x)$ is improper fuzzy Riemann-integrable on $[a, \infty)$ and the improper fuzzy Riemann-integrable is a fuzzy number. Furthermore, we have

$$
\int_{a}^{\infty} \widetilde{f}(x) d x=\left[\int_{a}^{\infty} \underline{f}_{\alpha}(x) d x, \int_{a}^{\infty} \bar{f}_{\alpha}(x) d x\right] .
$$

H-difference of fuzzy numbers is defined as follows.

Definition 2.6. If $\widetilde{u}, \widetilde{v} \in \mathcal{F}(\mathbb{R})$ and if there exists a fuzzy subset $\xi \in \mathcal{F}(\mathbb{R})$ such that $\xi+\widetilde{u}=\widetilde{v}$, then $\xi$ is unique. In this case, $\xi$ is called the Hukuhara difference, or simply H-difference of $u$ and $v$ and is denoted by $\widetilde{v}-\mathrm{H} \widetilde{u}$.

In the next definition, the strongly generalized differentiability concept is provided.

Definition $2.7([12,13])$. Let $\tilde{f}:(a, b) \rightarrow \mathcal{F}(\mathbb{R})$ and $x_{0} \in(a, b)$. We say that $\tilde{f}$ is strongly generalized differentiable at $x_{0}$, if there exists an element $\widetilde{f}^{\prime}\left(x_{0}\right) \in \mathcal{F}(\mathbb{R})$, such that

1. for all $h>0$ sufficiently small, there exist $\widetilde{f}\left(x_{0}+h\right)-H \widetilde{f}\left(x_{0}\right), \widetilde{f}\left(x_{0}\right)-H \widetilde{f}\left(x_{0}-h\right)$, and the limits (in the metric D)

$$
\lim _{h \rightarrow 0} \frac{\widetilde{f}\left(x_{0}+h\right)-H \widetilde{f}\left(x_{0}\right)}{h}=\lim _{h \rightarrow 0} \frac{\widetilde{f}\left(x_{0}\right)-H \widetilde{f}\left(x_{0}-h\right)}{h}=\widetilde{f}^{\prime}\left(x_{0}\right)
$$

or,

2. for all $h>0$ sufficiently small, there exist $\widetilde{f}\left(x_{0}\right)-H \widetilde{f}\left(x_{0}+h\right), \widetilde{f}\left(x_{0}-h\right)-H \tilde{f}\left(x_{0}\right)$, and the limits (in the metric D)

$$
\lim _{h \rightarrow 0} \frac{\widetilde{\mathbf{f}}\left(x_{0}\right)-H \widetilde{f}\left(x_{0}+h\right)}{-h}=\lim _{h \rightarrow 0} \frac{\widetilde{\mathbf{f}}\left(x_{0}-h\right)-H \widetilde{f}\left(x_{0}\right)}{-h}=\widetilde{f}^{\prime}\left(x_{0}\right) .
$$


In this paper, we denote the space of all continuous fuzzy functions on $[a, b] \subseteq \mathbb{R}$ and the space of all Lebesgue integrable fuzzy functions on the bounded interval $[a, b]$ by $\mathbb{C}^{\mathcal{F}}[a, b]$ and $L^{\mathcal{F}}[a, b]$, respectively.

Definition 2.8 ([38]). Let $\tilde{f} \in \mathbb{C}^{\mathcal{F}}[a, b] \cap \mathrm{L}^{\mathcal{F}}[a, b]$ be a fuzzy function. The fuzzy Riemann-Liouville integral of the fuzzy function $\tilde{f}$ is defined as follows.

$$
\left(I^{\beta} \tilde{f}\right)(x)=\frac{1}{\Gamma(\beta)} \int_{0}^{x} \frac{\tilde{f}(t)}{(x-t)^{1-\beta}} d t, \quad x, \beta \in \mathbb{R}_{+} .
$$

Theorem 2.9 ([10]). Let $\tilde{\mathrm{f}} \in \mathbb{C}^{\mathcal{F}}[\mathrm{a}, \mathrm{b}] \cap \mathrm{L}^{\mathcal{F}}[\mathrm{a}, \mathrm{b}]$ be a fuzzy function. The fuzzy Riemann-Liouville integral of the fuzzy function $\widetilde{\mathrm{f}}$ is as follows.

$$
\left[\left(I^{\beta} \tilde{f}\right)(x)\right]_{\alpha}=\left[I^{\beta} \underline{f}_{\alpha}(x), I^{\beta} \bar{f}_{\alpha}(x)\right], \quad 0 \leqslant \alpha \leqslant 1,
$$

where

$$
\begin{array}{ll}
\left(\mathrm{I}^{\beta} \underline{f}_{\alpha}\right)(x)=\frac{1}{\Gamma(\beta)} \int_{0}^{x} \frac{\underline{f}_{\alpha}(t)}{(x-t)^{1-\beta}} d t, \quad x, \beta \in \mathbb{R}_{+}, \\
\left(\mathrm{I}^{\beta} \bar{f}_{\alpha}\right)(x)=\frac{1}{\Gamma(\beta)} \int_{0}^{x} \frac{\bar{f}_{\alpha}(t)}{(x-t)^{1-\beta}} d t, \quad x, \beta \in \mathbb{R}_{+} .
\end{array}
$$

\subsection{Caputo fuzzy fractional derivative}

In this subsection, we provide some definitions and theorems on Caputo fuzzy fractional derivative. In [36], the authors extended the Caputo fractional derivative of crisp case into fuzzy setting. Here, we provide some of the concepts proposed.

Lemma 2.10 ([36]). Let $\mathrm{f}(\mathrm{x})$ be a crisp continuous function and ( $\lceil\beta\rceil)$-times differentiable in the independent variable $x$ over the interval of differentiation (integration) $[0, x]$. Then the relation

$$
{ }^{C} D^{\beta} f(x)={ }^{R L} D^{\beta}\left(f(x)-\sum_{k=0}^{\lceil\beta\rceil} \frac{x^{k}}{k !} f_{0}^{(k)}\right), \quad \beta \in(n-1, n], n \in \mathbb{N},
$$

holds, where $f_{0}^{(k)}=\left.\frac{d^{k} f(x)}{d x^{k}}\right|_{x=0}$ and ${ }^{C} D^{\beta}$ denotes Caputo derivative operator, while $\lceil\beta\rceil$ and $\lfloor\beta\rfloor$ are the value $\beta$ rounded up and down to the closest integer number, respectively. ${ }^{\mathrm{R}} \mathrm{D}^{\beta}$ is the common Riemann-Liouville fractional derivative operator which is defined as follows

$$
{ }^{R L} D^{\beta} f(x)=\frac{1}{\Gamma(\lceil\beta\rceil-\beta)} \frac{d^{\lceil\beta\rceil}}{d x\lceil\beta\rceil} \int_{0}^{x} \frac{f(t)}{(x-t)^{1-\lceil\beta\rceil+\beta}} d t .
$$

Definition 2.11 ([36]). Let $\widetilde{f}(x) \in \mathbb{C}^{\mathcal{F}}[0, b] \cap \mathrm{L}^{\mathcal{F}}[0, b], \widetilde{G}(x)=\frac{1}{\Gamma(\lceil\beta\rceil-\beta)} \int_{0}^{x} \frac{\widetilde{f}(t)-\sum_{k=0}^{[\beta]} \frac{t^{k}}{k !} \widetilde{f}_{0}^{(k)}}{(x-t)^{1-|\beta|+\beta}} d t$, and

$$
\widetilde{\mathrm{H}}\left(x_{0}\right)=\lim _{h \rightarrow 0^{+}} \frac{\widetilde{\mathrm{G}}\left(x_{0}+h\right) \ominus \widetilde{\mathrm{G}}\left(x_{0}\right)}{\mathrm{h}}=\lim _{h \rightarrow 0^{+}} \frac{\widetilde{\mathrm{G}}\left(x_{0}\right) \ominus \widetilde{\mathrm{G}}\left(x_{0}-\mathrm{h}\right)}{\mathrm{h}}
$$

and

$$
\widetilde{\mathrm{L}}\left(x_{0}\right)=\lim _{\mathrm{h} \rightarrow 0^{+}} \frac{\widetilde{\mathrm{G}}\left(x_{0}\right) \ominus \widetilde{\mathrm{G}}\left(x_{0}+\mathrm{h}\right)}{-\mathrm{h}}=\lim _{\mathrm{h} \rightarrow 0^{+}} \frac{\widetilde{\mathrm{G}}\left(x_{0}-\mathrm{h}\right) \ominus \widetilde{\mathrm{G}}\left(x_{0}\right)}{-\mathrm{h}} .
$$

$\widetilde{f}(x)$ is Caputo fuzzy fractional differentiable function of order $0<\beta \leqslant 1$, if there exists an element ${ }^{C} D^{\beta} \widetilde{f}\left(x_{0}\right) \in \mathbb{C}^{\mathcal{F}}$ such that for all $0 \leqslant \alpha \leqslant 1$ and for $h>0$ sufficiently near zero, either, 
1. ${ }^{c} D^{\beta} \widetilde{f}\left(x_{0}\right)=\lim _{h \rightarrow 0^{+}} \frac{\widetilde{G}\left(x_{0}+h\right) \ominus \widetilde{G}\left(x_{0}\right)}{h}=\lim _{h \rightarrow 0^{+}} \frac{\widetilde{G}\left(x_{0}\right) \ominus \widetilde{G}\left(x_{0}-h\right)}{h}$, or

2. ${ }^{C} D^{\beta} \widetilde{f}\left(x_{0}\right)=\lim _{h \rightarrow 0^{+}} \frac{\widetilde{G}\left(x_{0}\right) \ominus \widetilde{G}\left(x_{0}+h\right)}{-h}=\lim _{h \rightarrow 0^{+}} \frac{\widetilde{G}\left(x_{0}-h\right) \ominus \widetilde{G}\left(x_{0}\right)}{-h}$, for $0<\beta \leqslant 1$.

If the fuzzy function $\widetilde{f}(x)$ is differentiable as in Definition $2.11(1)$, it is called Caputo fuzzy fractional differentiable in the first form. If $\widetilde{f}(x)$ is differentiable as in Definition 2.11 (2), it is called Caputo fuzzy fractional differentiable in the second form.

Theorem 2.12 ([36]). Let $\widetilde{\mathrm{f}}(\mathrm{x}) \in \mathbb{C}^{\mathcal{F}}[0, \mathrm{~b}] \cap \mathrm{L}^{\mathcal{F}}[0, \mathrm{~b}]$ be a fuzzy function and $[\widetilde{\mathrm{f}}(\mathrm{x})]_{\alpha}=\left[\underline{\mathrm{f}}_{\alpha}(\mathrm{x}), \overline{\mathrm{f}}_{\alpha}(\mathrm{x})\right]$ for $\alpha \in[0,1]$ and $\mathrm{x}_{0} \in(0, \mathrm{~b})$. Then

1. If $\widetilde{\mathbf{f}}(\mathrm{x})$ is Caputo fuzzy fractional differentiable in the first form, then for every $0<\beta \leqslant 1$,

$$
\left[{ }^{C} D^{\beta} \widetilde{f}\left(x_{0}\right)\right]_{\alpha}=\left[{ }^{C} D^{\beta} \underline{f}_{\alpha}\left(x_{0}\right),{ }^{C} D^{\beta} \bar{f}_{\alpha}\left(x_{0}\right)\right] .
$$

2. If $\widetilde{\mathrm{f}}(\mathrm{x})$ is Caputo fuzzy fractional differentiable in the second form, then for every $0<\beta \leqslant 1$,

$$
\left[{ }^{\mathrm{C}} \mathrm{D}^{\beta} \widetilde{\mathrm{f}}\left(\mathrm{x}_{0}\right)\right]_{\alpha}=\left[{ }^{\mathrm{C}} \mathrm{D}^{\beta} \overline{\mathrm{f}}_{\alpha}\left(\mathrm{x}_{0}\right),{ }^{\mathrm{C}} \mathrm{D}^{\beta} \underline{\underline{f}}_{\alpha}\left(x_{0}\right)\right],
$$

where

$$
\begin{aligned}
{ }^{C} D^{\beta} \underline{f}_{\alpha}\left(x_{0}\right) & =\left[\frac{1}{\Gamma(\lceil\beta\rceil-\beta)} \int_{0}^{x} \frac{D^{\lceil\beta\rceil} \underline{f}_{\alpha}(t)}{(x-t)^{1-\lceil\beta\rceil+\beta}}\right]_{x=x_{0}}, \\
{ }^{C} D^{\beta} \bar{f}_{\alpha}\left(x_{0}\right) & =\left[\frac{1}{\Gamma(\lceil\beta\rceil-\beta)} \int_{0}^{x} \frac{D^{\lceil\beta\rceil} \bar{f}_{\alpha}(t)}{(x-t)^{1-\lceil\beta\rceil+\beta}}\right]_{x=x_{0}}, \\
D^{k} f(t) & =\frac{d^{k} f(t)}{d t^{k}} .
\end{aligned}
$$

Next, we give the definition for the classical Sumudu transform when dealing with Caputo's fractional derivative of crisp type.

Definition 2.13 ([19, 24]). The classical Sumudu transform for Caputo's fractional derivative of function $f$ is given by

$$
s\left[{ }^{C} D^{\beta} f(x)\right](u)=u^{-\beta} G(u)-\sum_{k=0}^{n-1} \frac{f^{k}(0)}{u^{\beta-k}}, \quad \beta \in(n-1, n] .
$$

Since in this paper, we only consider $0<\beta<1$, Definition 2.13 can be simplified as follows.

$$
s\left[{ }^{C} D^{\beta} f(x)\right](u)=\frac{G(u)-f^{0}(0)}{u^{\beta}}, \quad \beta \in(0,1] .
$$

Note that when $\beta=1$, the definition is similar to the definition of Sumudu transform for first order derivative.

\section{Fuzzy Sumudu transform for Caputo fuzzy fractional derivative}

In this part, we recall the definition of FST and later we propose a new result on the property of FST for Caputo fuzzy fractional derivative.

Definition 3.1 ([1, 4]). Let $\tilde{f}: \mathbb{R} \rightarrow \mathcal{F}(\mathbb{R})$ be a continuous fuzzy function. Suppose $\widetilde{f}(u x) \odot e^{-x}$ is improper fuzzy Riemann-integrable on $[0, \infty)$, then $\int_{0}^{\infty} \tilde{f}(u x) \odot e^{-x} d x$ is called fuzzy Sumudu transform and is denoted by

$$
G(u)=S[\tilde{f}(x)](u)=\int_{0}^{\infty} \tilde{f}(u x) \odot e^{-x} d x, \quad u \in\left[-\tau_{1}, \tau_{2}\right],
$$

where the variable $u$ is used to factor the variable $x$ in the argument of the fuzzy function and $\tau_{1}, \tau_{2}>0$. 
The FST can also be written into the following parametric form.

$$
\mathcal{S}[\widetilde{f}(x)](u)=\left[s\left[f_{\alpha}(x)\right](u), s\left[\bar{f}_{\alpha}(x)\right](u)\right] .
$$

In the following theorem, we introduce a new property of FST for Caputo fuzzy fractional derivative. This is done by directly extending the definition for classical Sumudu transform of Caputo fractional derivative into fuzzy setting.

Theorem 3.2. Let $\widetilde{\mathrm{f}}(\mathrm{x}) \in \mathbb{C}^{\mathcal{F}}[0, \mathrm{~b}] \cap \mathrm{L}^{\mathcal{F}}[0, \mathrm{~b}]$ be a continuous fuzzy function, and ${ }^{\mathrm{C}} \mathrm{D}^{\beta} \widetilde{\mathrm{f}}$ is the Caputo fuzzy fractional derivative of $\tilde{\mathrm{f}}$ on $[0, \infty)$. Then, for $0<\beta \leqslant 1$, we have

$$
\mathcal{S}\left[{ }^{\mathrm{C}} \mathrm{D}^{\beta} \widetilde{\mathrm{f}}(\mathrm{x})\right](\mathrm{u})=\frac{\mathrm{G}(\mathrm{u})-{ }^{\mathrm{H}} \tilde{\mathrm{f}}\left(\mathrm{x}_{0}\right)}{\mathrm{u}^{\beta}},
$$

where $\tilde{\mathrm{f}}$ is Caputo fuzzy fractional differentiable in the first form, or

$$
\mathcal{S}\left[{ }^{\mathrm{C}} D^{\beta} \tilde{\mathrm{f}}(\mathrm{x})\right](\mathrm{u})=\frac{-\widetilde{\mathrm{f}}\left(\mathrm{x}_{0}\right)-{ }^{\mathrm{H}}(-\mathrm{G}(\mathrm{u}))}{\mathrm{u}^{\beta}},
$$

where $\tilde{\mathrm{f}}$ is Caputo fuzzy fractional differentiable in the second form.

Proof. First, we assume $\tilde{\mathrm{f}}$ is Caputo fuzzy fractional differentiable in the first form (Theorem 2.12 (1)). Therefore,

$$
\frac{G(u)-H \tilde{f}(0)}{u^{\beta}}=\left[\frac{s[\underline{f}(x)](u)-\underline{f}(0)}{u^{\beta}}, \frac{s[\bar{f}(x)](u)-\bar{f}(0)}{u^{\beta}}\right] .
$$

From the classical Sumudu transform for Caputo fractional derivative, we know that

$$
s\left[{ }^{C} D^{\beta} \underline{f}(x)\right](u)=\frac{s[\underline{f}(x)](u)-\underline{f}(0)}{u^{\beta}} \text { and } s\left[{ }^{C} D^{\beta} \bar{f}(x)\right](u)=\frac{s[\bar{f}(x)](u)-\bar{f}(0)}{u^{\beta}} .
$$

Then,

$$
\frac{G(u)-{ }^{H} \tilde{f}(0)}{u^{\beta}}=\left[s\left[{ }^{C} D^{\beta} \underline{f}(x)\right](u), s\left[{ }^{C} D^{\beta} \bar{f}(x)\right](u)\right] .
$$

Since $\tilde{f}$ is Caputo fuzzy fractional differentiable in the first form

$$
\frac{G(u)-H \widetilde{f}\left(x_{0}\right)}{u^{\beta}}=\mathcal{S}\left[{ }^{C} D^{\beta} \widetilde{f}(x)\right](u) .
$$

Now, we assume that $\tilde{f}$ is Caputo fuzzy fractional differentiable in the second form (Theorem 2.12 (2)). Therefore,

$$
\frac{-\tilde{f}\left(x_{0}\right)-{ }^{H}(-G(u))}{u^{\beta}}=\left[\frac{-\bar{f}\left(x_{0}\right)-(-s[\bar{f}(x)](u))}{u^{\beta}}, \frac{-\underline{f}\left(x_{0}\right)-(-s[\mathfrak{f}(x)](u))}{u^{\beta}}\right] .
$$

This is analogous to

$$
\frac{-\widetilde{f}\left(x_{0}\right)-H(-G(u))}{u^{\beta}}=\left[\frac{s[\bar{f}(x)](u)-\bar{f}(0)}{u^{\beta}}, \frac{s[\underline{f}(x)](u)-\underline{f}(0)}{u^{\beta}}\right] .
$$

From the classical Sumudu transform for Caputo fractional derivative, finally we have

$$
\frac{-\widetilde{f}\left(x_{0}\right)-{ }^{H}(-G(u))}{u^{\beta}}=\left[s\left[{ }^{C} D^{\beta} \bar{f}(x)\right](u), s\left[{ }^{C} D^{\beta} \underline{f}(x)\right](u)\right] .
$$

Since $\tilde{f}$ is Caputo fuzzy fractional differentiable in the second form, then we finally have

$$
\frac{-\widetilde{f}\left(x_{0}\right)-{ }^{H}(-G(u))}{u^{\beta}}=\mathcal{S}\left[{ }^{C} D^{\beta} \widetilde{f}(x)\right](u) .
$$

The proof is complete. 


\section{Procedures for solving linear FFDEs using FST}

Consider the following linear FFDE.

$$
\left\{\begin{array}{l}
{ }^{C} D^{\beta} \widetilde{y}(x)=f[x, \widetilde{y}(x)], \\
\widetilde{y}_{\alpha}\left(x_{0}\right)=\left[\underline{y}_{\alpha}(0), \bar{y}_{\alpha}(0)\right],
\end{array}\right.
$$

where $f \in \mathbb{C}^{\mathcal{F}}(a, b) \cap \mathrm{L}^{\mathcal{F}}(a, b)$ and $x_{0} \in(a, b)$. By using FST on both sides of Eq. (4.1), we have

$$
\mathcal{S}\left[{ }^{C} D^{\beta} \widetilde{y}(x)\right](u)=\mathcal{S}[f(x, \widetilde{y}(x))](u) .
$$

Case 1 : If we consider $\widetilde{y}$ to be Caputo fuzzy fractional differentiable in the first form, then from Theorem 2.12 (1), we get $\left[{ }^{C} D^{\beta} \widetilde{y}\left(x_{0}\right)\right]_{\alpha}=\left[{ }^{C} D^{\beta} \underline{y}_{\alpha}\left(x_{0}\right),{ }^{C} D^{\beta} \bar{y}_{\alpha}\left(x_{0}\right)\right]$. Now, we obtain the following system

$$
\begin{cases}{ }^{C} D^{\beta} \underline{y}_{\alpha}(x)=f[x, \widetilde{y}(x)]=\underline{f}_{\alpha}[x, \widetilde{y}(x)], & \underline{y}_{\alpha}\left(x_{0}\right)=\underline{y}_{\alpha}(0), \\ { }^{C} D^{\beta} \bar{y}_{\alpha}(x)=\overline{\bar{f}[x, \widetilde{y}(x)]}=\bar{f}_{\alpha}[x, \widetilde{y}(x)], & \bar{y}_{\alpha}\left(x_{0}\right)=\bar{y}_{\alpha}(0),\end{cases}
$$

where $\beta \in(0,1]$. From Theorem 3.2, we have

$$
\mathcal{S}\left[{ }^{C} D^{\beta} \widetilde{y}(x)\right](u)=\frac{G(u)-{ }^{H} \widetilde{y}\left(t_{0}\right)}{u^{\beta}} .
$$

Therefore,

$$
\left\{\begin{array}{l}
s\left[\underline{f}_{\alpha}(x, \widetilde{y}(x))\right](u)=\frac{s\left[\underline{y}_{\alpha}(x)\right](u)-\underline{y}_{\alpha}(0)}{u}, \\
s\left[\bar{f}_{\alpha}(x, \widetilde{y}(x))\right](u)=\frac{s\left[\bar{y}_{\alpha}(x)\right](u)-\bar{y}_{\alpha}(0)}{u^{\beta}}
\end{array}\right.
$$

where,

$$
\underline{f}_{\alpha}(x, \tilde{y}(x))=\min \left\{\tilde{\mathfrak{f}}(x, u) \mid u \in\left[\underline{y}_{\alpha}(x), \bar{y}_{\alpha}(x)\right]\right\} \quad \text { and } \quad \bar{f}_{\alpha}(x, \widetilde{y}(x))=\max \left\{\tilde{f}(x, u) \mid u \in\left[\underline{y}_{\alpha}(x), \bar{y}_{\alpha}(x)\right]\right\} .
$$

To solve Eq. (4.2), first we assume that

$$
s\left[\underline{y}_{\alpha}(x)\right](u)=\mathrm{L}_{\alpha}^{1}(u), \quad s\left[\bar{y}_{\alpha}(x)\right](u)=\mathrm{U}_{\alpha}^{1}(u) .
$$

$L_{\alpha}^{1}(u)$ and $U_{\alpha}^{1}(u)$ are the solutions of Eq. (4.2) under this case. We obtain $\underline{y}_{\alpha}(x)$ and $\bar{y}_{\alpha}(x)$ using the inverse FST as the following

$$
\underline{y}_{\alpha}(x)=s^{-1}\left[L_{\alpha}^{1}(u)\right], \quad \bar{y}_{\alpha}(x)=s^{-1}\left[U_{\alpha}^{1}(u)\right] .
$$

Case 2 : If we consider $\widetilde{y}$ to be Caputo fuzzy fractional differentiable in the second form, then from Theorem 2.12 (2), we get $\left[{ }^{C} D^{\beta} \widetilde{y}\left(x_{0}\right)\right]_{\alpha}=\left[{ }^{C} D^{\beta} \bar{y}_{\alpha}\left(x_{0}\right),{ }^{C} D^{\beta} \underline{y}_{\alpha}\left(x_{0}\right)\right]$. Now, we obtain the following system

$$
\begin{cases}{ }^{C} D^{\beta} \underline{y}_{\alpha}(x)=\overline{f[x, \widetilde{y}(x)]}=\bar{f}_{\alpha}[x, \widetilde{y}(x)], & \underline{y}_{\alpha}\left(x_{0}\right)=\underline{y}_{\alpha}(0), \\ { }^{C} D^{\beta} \bar{y}_{\alpha}(x)=\underline{f[x, \widetilde{y}(x)]}=\underline{f}_{\alpha}[x, \widetilde{y}(x)], & \bar{y}_{\alpha}\left(x_{0}\right)=\bar{y}_{\alpha}(0),\end{cases}
$$

where $\beta \in(0,1]$. From Theorem 3.2,

$$
\mathcal{S}\left[{ }^{C} D^{\beta} \widetilde{y}(x)\right](u)=\frac{-\widetilde{y}\left(t_{0}\right)-{ }^{H}(-G(u))}{u^{\beta}} .
$$

Therefore,

$$
\left\{\begin{array}{l}
s\left[\underline{f}_{\alpha}(x, \widetilde{y}(x))\right](u)=\frac{s\left[\underline{y}_{\alpha}(x)\right](u)-\underline{y}_{\alpha}(0)}{u \beta}, \\
s\left[\bar{f}_{\alpha}(x, \widetilde{y}(x))\right](u)=\frac{s\left[\bar{y}_{\alpha}(x)\right](u)-\bar{y}_{\alpha}(0)}{u^{\beta}},
\end{array}\right.
$$

where, 


$$
\underline{f}_{\alpha}(x, \widetilde{y}(x))=\min \left\{\tilde{f}(x, u) \mid u \in\left[\underline{y}_{\alpha}(x), \bar{y}_{\alpha}(x)\right]\right\} \quad \text { and } \quad \bar{f}_{\alpha}(x, \widetilde{y}(x))=\max \left\{\tilde{f}(x, u) \mid u \in\left[\underline{y}_{\alpha}(x), \bar{y}_{\alpha}(x)\right]\right\} .
$$

To solve Eq. (4.3), first we assume that

$$
s\left[\underline{y}_{\alpha}(x)\right](u)=\mathrm{L}_{\alpha}^{2}(u), \quad s\left[\bar{y}_{\alpha}(x)\right](u)=\mathrm{U}_{\alpha}^{2}(u) .
$$

$\mathrm{L}_{\alpha}^{2}(\mathrm{u})$ and $\mathrm{U}_{\alpha}^{2}(\mathrm{u})$ are the solutions of Eq. (4.3) for this case. We have $\underline{y}_{\alpha}(x)$ and $\bar{y}_{\alpha}(x)$ by the inverse of FST as the following

$$
\underline{y}_{\alpha}(x)=s^{-1}\left[L_{\alpha}^{2}(u)\right], \quad \bar{y}_{\alpha}(x)=s^{-1}\left[\mathrm{U}_{\alpha}^{2}(u)\right] .
$$

\section{A numerical example}

In this part, the method proposed will be demonstrated on a linear FFDE. This is to show that the method is practicable.

Example 5.1. The following linear FFDE is considered.

$$
\left\{\begin{array}{l}
{ }^{C} D^{\beta} \widetilde{y}(x)=\widetilde{y}(x), \\
\widetilde{y}\left(x_{0}\right)=\left[\underline{y}_{\alpha}(0), \bar{y}_{\alpha}(0)\right] .
\end{array}\right.
$$

Case 1: By taking fuzzy Sumudu transform on both sides of (5.1), we have

$$
\mathcal{S}\left[{ }^{\mathrm{C}} \mathrm{D}^{\beta} \widetilde{\mathrm{y}}(\mathrm{x})\right](\mathrm{u})=\mathcal{S}[\widetilde{\mathrm{y}}(\mathrm{x})](\mathrm{u}) .
$$

From Theorem 2.12 (1) for Caputo fuzzy fractional differentiability in the first form, we have

$$
\left[{ }^{C} D^{\beta} \widetilde{y}\left(x_{0}\right)\right]_{\alpha}=\left[{ }^{C} D^{\beta} \underline{y}_{\alpha}\left(x_{0}\right),{ }^{C} D^{\beta} \bar{y}_{\alpha}\left(x_{0}\right)\right],
$$

and from Theorem 3.2,

$$
\mathcal{S}\left[{ }^{C} D^{\beta} \widetilde{y}(x)\right](u)=\frac{G(u)-{ }^{H} \widetilde{y}\left(x_{0}\right)}{u^{\beta}}
$$

we have

$$
\left\{\begin{array}{l}
s\left[\underline{y}_{\alpha}(x)\right](u)=\frac{s\left[\underline{y}_{\alpha}(x)\right](u)-\underline{y}_{\alpha}(0)}{u^{\beta}}, \\
s\left[\bar{y}_{\alpha}(x)\right](u)=\frac{s\left[\bar{y}_{\alpha}(x)\right](u)-\bar{y}_{\alpha}(0)}{u^{\beta}} .
\end{array}\right.
$$

Then, we obtain

$$
\left\{\begin{array}{l}
\left(1-u^{\beta}\right) s\left[\underline{y}_{\alpha}(x)\right](u)=\underline{y}_{\alpha}(0), \\
\left(1-u^{\beta}\right) s\left[\bar{y}_{\alpha}(x)\right](u)=\bar{y}_{\alpha}(0) .
\end{array}\right.
$$

By applying inverse Sumudu transform, we obtain

$$
\left\{\begin{array}{l}
\underline{y}_{\alpha}(x)=\underline{y}_{\alpha}(0) s^{-1}\left[\frac{1}{1-u^{\beta}}\right], \\
\bar{y}_{\alpha}(x)=\bar{y}_{\alpha}(0) s^{-1}\left[\frac{1}{1-u^{\beta}}\right] .
\end{array}\right.
$$

By relation,

$$
s\left[x^{\gamma-1} E_{\beta, \gamma}\left(\mp \chi^{\beta}\right)\right](u)=\frac{u^{\gamma-1}}{1 \pm u^{\beta}}
$$


finally, we have

$$
\left\{\begin{array}{l}
\underline{y}_{\alpha}(x)=\underline{y}_{\alpha}(0) E_{\beta, 1}\left[x^{\beta}\right], \\
\bar{y}_{\alpha}(x)=\bar{y}_{\alpha}(0) E_{\beta, 1}\left[x^{\beta}\right] .
\end{array}\right.
$$

$\mathrm{E}_{\beta, 1}\left[\mathrm{x}^{\beta}\right]$ is the Mittag-Leffler function defined by

$$
\mathrm{E}_{\beta, 1}\left[x^{\beta}\right]=\sum_{\mathrm{k}=0}^{\infty} \frac{\left(x^{\beta}\right)^{\mathrm{k}}}{\Gamma(\beta \mathrm{k}+1)} .
$$

Case 2: Using fuzzy Sumudu transform on Eq. (5.1), we have

$$
\mathcal{S}\left[{ }^{\mathrm{C}} \mathrm{D}^{\beta} \widetilde{\mathrm{y}}(\mathrm{x})\right](\mathrm{u})=\mathcal{S}[\widetilde{\mathrm{y}}(\mathrm{x})](\mathrm{u}) .
$$

From Theorem 2.12 (2) for Caputo fuzzy fractional differentiability in the second form,

$$
\left[{ }^{C} D^{\beta} \widetilde{y}\left(x_{0}\right)\right]_{\alpha}=\left[{ }^{C} D^{\beta} \bar{y}_{\alpha}\left(x_{0}\right),{ }^{C} D^{\beta} \underline{y}_{\alpha}\left(x_{0}\right)\right],
$$

and from Theorem 3.2

$$
\mathcal{S}\left[{ }^{C} D^{\beta} \widetilde{f}(x)\right](u)=\frac{-\widetilde{f}\left(x_{0}\right)-{ }^{H}(-G(u))}{u^{\beta}},
$$

we have

$$
\left\{\begin{array}{l}
s\left[\underline{y}_{\alpha}(x)\right](u)=\frac{-\bar{y}_{\alpha}(0)-\left[-s\left[\bar{y}_{\alpha}(x)\right](u)\right]}{u^{\beta}}, \\
s\left[\bar{y}_{\alpha}(x)\right](u)=\frac{-\underline{y}_{\alpha}(0)-\left[-s\left[\underline{y}_{\alpha}(x)\right](u)\right]}{u^{\beta}},
\end{array}\right.
$$

equivalent to

$$
\left\{\begin{array}{l}
s\left[\underline{y}_{\alpha}(x)\right](u)=\frac{s\left[\bar{y}_{\alpha}(x)\right](u)-\bar{y}_{\alpha}(0)}{u^{\beta}}, \\
s\left[\bar{y}_{\alpha}(x)\right](u)=\frac{s\left[\underline{y}_{\alpha}(x)\right](u)-\underline{y}_{\alpha}(0)}{u^{\beta}} .
\end{array}\right.
$$

Then, we obtain

$$
\left\{\begin{array}{l}
\left(1+u^{2 \beta}\right) s\left[\underline{y}_{\alpha}(x)\right](u)=\underline{y}_{\alpha}(0)-u^{\beta} \bar{y}_{\alpha}(0), \\
\left(1+u^{2 \beta}\right) s\left[\bar{y}_{\alpha}(x)\right](u)=\bar{y}_{\alpha}(0)-u^{\beta} \underline{y}_{\alpha}(0) .
\end{array}\right.
$$

By applying inverse Sumudu transform, we have

$$
\left\{\begin{array}{l}
\underline{y}_{\alpha}(x)=\underline{y}_{\alpha}(0) s^{-1}\left[\frac{1}{1+u^{2 \beta}}\right]-\bar{y}_{\alpha}(0) s^{-1}\left[\frac{u^{\beta}}{1+u 2^{\beta}}\right], \\
\bar{y}_{\alpha}(x)=\bar{y}_{\alpha}(0) s^{-1}\left[\frac{1}{1+u^{2 \beta}}\right]-\underline{y}_{\alpha}(0) s^{-1}\left[\frac{u^{\beta}}{1+u 2^{\beta}}\right] .
\end{array}\right.
$$

By relation,

finally, we have

$$
s\left[x^{\gamma-1} E_{\beta, \gamma}\left(\mp x^{\beta}\right)\right](u)=\frac{u^{\gamma-1}}{1- \pm u^{\beta}},
$$

$$
\left\{\begin{array}{l}
\underline{y}_{\alpha}(x)=\underline{y}_{\alpha}(0) E_{2 \beta, 1}\left[-x^{2 \beta}\right]-\bar{y}_{\alpha}(0) x^{\beta} E_{2 \beta, \beta+1}\left[-\chi^{2 \beta}\right], \\
\bar{y}_{\alpha}(x)=\bar{y}_{\alpha}(0) E_{2 \beta, 1}\left[-x^{2 \beta}\right]-\underline{y}_{\alpha}(0) x^{\beta} E_{2 \beta, \beta+1}\left[-\chi^{2 \beta}\right],
\end{array}\right.
$$

where,

$$
E_{2 \beta, 1}\left[-\chi^{2 \beta}\right]=\sum_{k=0}^{\infty} \frac{\left(-\chi^{2 \beta}\right)^{k}}{\Gamma(2 \beta k+1)} \quad \text { and } \quad E_{2 \beta, \beta+1}\left[-\chi^{2 \beta}\right]=\sum_{k=0}^{\infty} \frac{\left(-\chi^{2 \beta}\right)^{k}}{\Gamma(2 \beta k+\beta+1)^{\prime}},
$$

are the Mittag-Leffler functions. 
Assume that $\widetilde{y}\left(x_{0}\right)=[1+\alpha, 3-\alpha]$. The numerical solutions of (5.1) for Cases 1 and 2 at $x=2$ are listed in Tables 1 and 2, respectively. For graphical results, please see in Figs. 1 and 2. Numerical solutions for both cases are obtained by expanding the Mittag-Leffler functions up to 11 terms. It can be concluded that the solutions of Eq. (5.1) are in agreement with the solutions of fuzzy differential equation as $\beta$ approaches to 1.

Table 1: Numerical solutions of Eq. (5.1) for Case 1 using different values of $\beta$.

\begin{tabular}{ccccccccc}
\hline \multicolumn{1}{c}{$\beta=0.4$} & \multicolumn{2}{c}{$\beta=0.6$} & \multicolumn{2}{c}{$\beta=0.8$} & \multicolumn{2}{c}{$\beta=1.0$} \\
\cline { 2 - 9 }$\alpha$ & $\underline{y}^{\beta}(x)$ & $\bar{y}^{\beta}(x)$ & $\underline{y}^{\beta}(x)$ & $\bar{y}^{\beta}(x)$ & $\underline{y}^{\beta}(x)$ & $\bar{y}^{\beta}(x)$ & $\underline{y}^{\beta}(x)$ & $\bar{y}^{\beta}(x)$ \\
\hline 0 & 16.5737 & 49.7212 & 12.0222 & 36.0667 & 9.1583 & 27.4749 & 7.3890 & 22.1670 \\
0.1 & 18.2311 & 48.0638 & 13.2244 & 34.8644 & 10.0741 & 26.5590 & 8.1279 & 21.4281 \\
0.2 & 19.8885 & 46.4064 & 14.4267 & 33.6622 & 10.9899 & 25.6432 & 8.8668 & 20.6892 \\
0.3 & 21.5459 & 44.7491 & 15.6289 & 32.4600 & 11.9058 & 24.7274 & 9.6057 & 19.9503 \\
0.4 & 23.2032 & 43.0917 & 16.8311 & 31.2578 & 12.8216 & 23.8116 & 10.3446 & 19.2114 \\
0.5 & 24.8606 & 41.4343 & 18.0333 & 30.0556 & 13.7374 & 22.8957 & 11.0835 & 18.4725 \\
0.6 & 26.5180 & 39.7770 & 19.2356 & 28.8533 & 14.6533 & 21.9799 & 11.8224 & 17.7336 \\
0.7 & 28.1753 & 38.1196 & 20.4378 & 27.6511 & 15.5691 & 21.0641 & 12.5613 & 16.9947 \\
0.8 & 29.8327 & 36.4622 & 21.6400 & 26.4489 & 16.4849 & 20.1482 & 13.3002 & 16.2558 \\
0.9 & 31.4901 & 34.8048 & 22.8422 & 25.2467 & 17.4008 & 19.2324 & 14.0391 & 15.5169 \\
1.0 & 33.1475 & 33.1475 & 24.0444 & 24.0444 & 18.3166 & 18.3166 & 14.7780 & 14.7780 \\
\hline
\end{tabular}

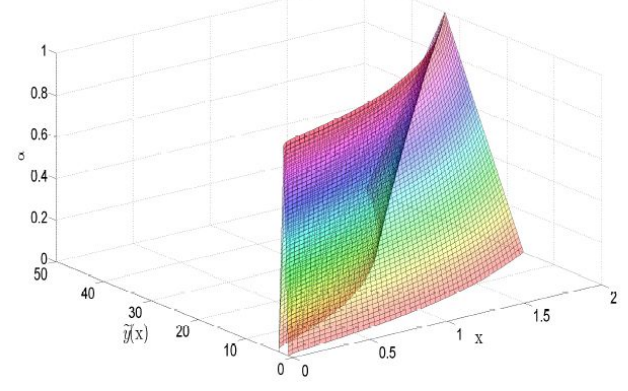

(a)

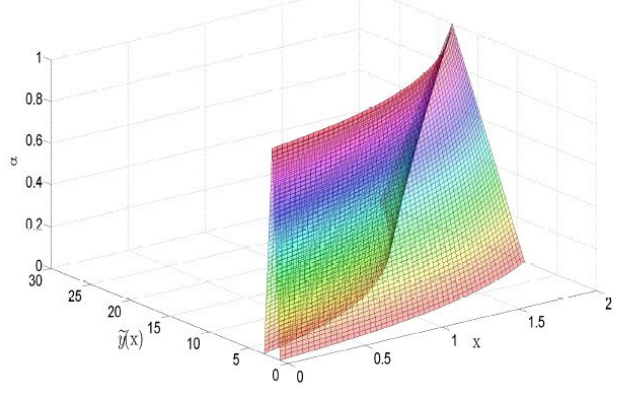

(c)

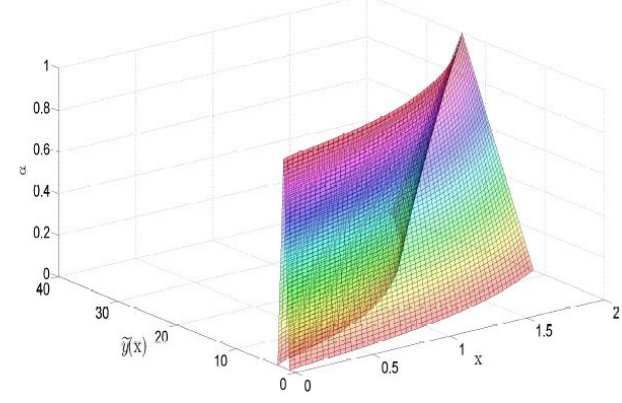

(b)

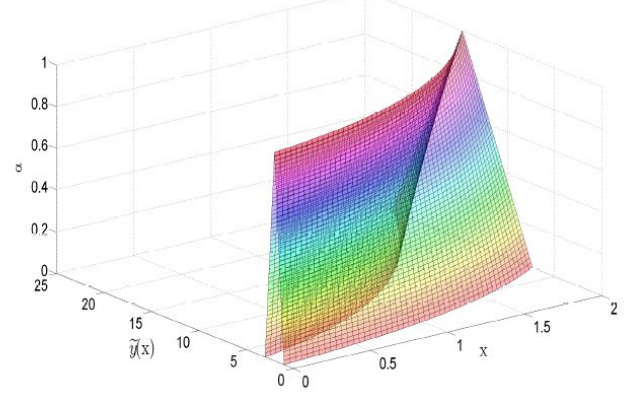

(d)

Figure 1: Numerical solutions of (5.1) for (a) $\beta=0.4$, (b) $\beta=0.6$, (c) $\beta=0.8$, and (d) $\beta=1$ (Case 1). 
Table 2: Numerical solutions of Eq. (5.1) for Case 2 using different values of $\beta$.

\begin{tabular}{ccccccccc}
\hline \multicolumn{1}{c}{$\beta=0.4$} & \multicolumn{2}{c}{$\beta=0.6$} & \multicolumn{2}{c}{$\beta=0.8$} & \multicolumn{2}{c}{$\beta=1.0$} \\
\cline { 2 - 9 }$\alpha$ & $\underline{y}^{\beta}(x)$ & $\bar{y}^{\beta}(x)$ & $\underline{y}^{\beta}(x)$ & $\bar{y}^{\beta}(x)$ & $\underline{y}^{\beta}(x)$ & $\bar{y}^{\beta}(x)$ & $\underline{y}^{\beta}(x)$ & $\bar{y}^{\beta}(x)$ \\
\hline 0 & -1.2888 & 0.1705 & -1.5531 & -0.4324 & -2.1398 & -1.2789 & -3.1440 & -2.1577 \\
0.1 & -1.2158 & 0.0976 & -1.4971 & -0.4884 & -2.0967 & -1.3219 & -3.0947 & -2.2071 \\
0.2 & -1.1428 & 0.0246 & -1.4410 & -0.5444 & -2.0537 & -1.3650 & -3.0454 & -2.2564 \\
0.3 & -1.0699 & -0.0484 & -1.3850 & -0.6005 & -2.0106 & -1.4080 & -2.9961 & -2.3057 \\
0.4 & -0.9969 & -0.1213 & -1.3289 & -0.6565 & -1.9676 & -1.4511 & -2.9468 & -2.3550 \\
0.5 & -0.9239 & -0.1943 & -1.2729 & -0.7125 & -1.9245 & -1.4941 & -2.8975 & -2.4043 \\
0.6 & -0.8510 & -0.2673 & -1.2169 & -0.7686 & -1.8815 & -1.5372 & -2.8481 & -2.4536 \\
0.7 & -0.7780 & -0.3402 & -1.1608 & -0.8246 & -1.8385 & -1.5802 & -2.7988 & -2.5029 \\
0.8 & -0.7051 & -0.4132 & -1.1048 & -0.8807 & -1.7954 & -1.6232 & -2.7495 & -2.5523 \\
0.9 & -0.6321 & -0.4862 & -1.0488 & -1.9367 & -1.7524 & -1.6663 & -2.7002 & -2.6016 \\
1.0 & -0.5591 & -0.5591 & -0.9927 & -0.9927 & -1.7093 & -1.7093 & -2.6509 & -2.6509 \\
\hline
\end{tabular}

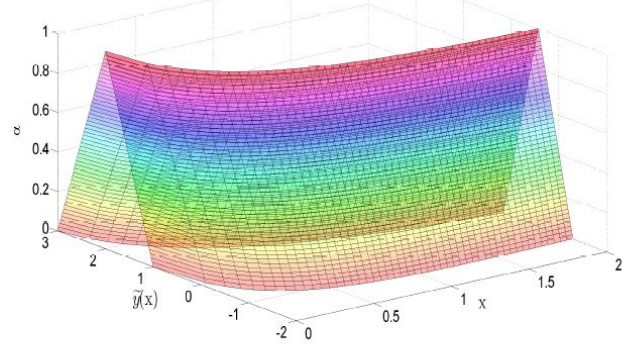

(a)

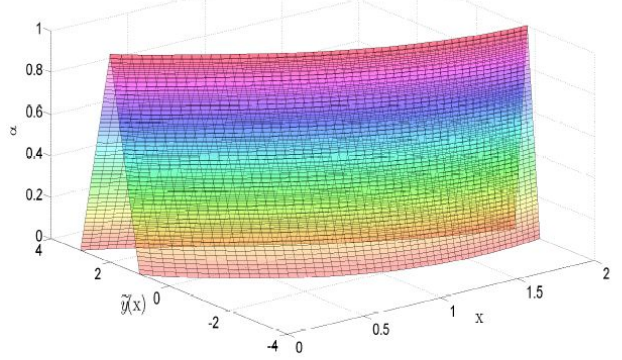

(c)

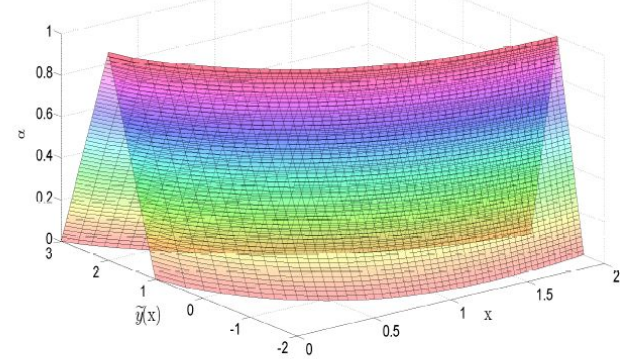

(b)

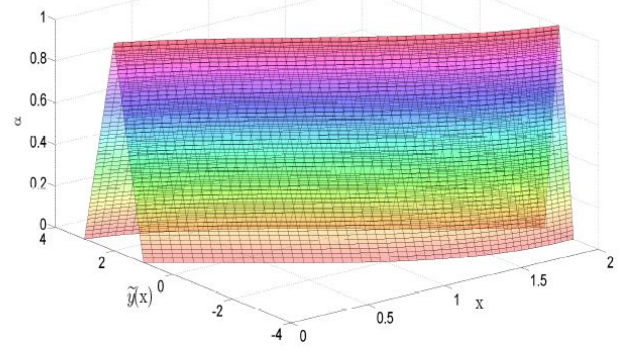

(d)

Figure 2: Numerical solutions of (5.1) for (a) $\beta=0.4$, (b) $\beta=0.6$, (c) $\beta=0.8$, and (d) $\beta=1$ (Case 2).

Remark 5.2. One of the disadvantages of the strongly generalized differentiability concept used in this paper is that a fuzzy fractional differential equation has no unique solution. That is the reason behind two cases that we solved. This disadvantage, from another point, is also an advantage as we can choose the singular point where the support of the solution changes its monotonicity. This means that we can obtain reversible solutions. Plus, we will also obtain stable and almost periodic solutions. Furthermore, researchers can choose the best solution from all the cases based on the problems faced. Further discussion 
on the advantages of strongly generalized differentiability can be seen in [11, 12]

\section{Conclusions}

In this paper, we have proposed a new analytical method for dealing with linear FFDEs involving Caputo fuzzy fractional derivatives. A new property of fuzzy Sumudu transform for Caputo fuzzy fractional derivative has been introduced. The new property has been used to construct a procedure for solving linear FFDEs. A numerical example has been solved to show that FST is functional. For future research, we intend to apply FST on nonlinear fuzzy differential equations of integer and fractional order. For that purpose, as fuzzy Sumudu transform cannot directly solve nonlinear problems, an integration with a numerical result has to be made. For example, an integration between fuzzy Sumudu transform and homotopy perturbation method. This will produce a new hybrid method, which then can be tested on nonlinear problems.

\section{Acknowledgment}

This research received funding by Ministry of Science, Technology and Innovation, Malaysia under the Fundamental Research Grant Scheme, project code 9003-00417.

\section{References}

[1] N. A. Abdul Rahman, M. Z. Ahmad, Applications of the fuzzy Sumudu transform for the solution of first order fuzzy differential equations, Entropy, 17 (2015), 4582-4601. 1, 3.1

[2] O. P. Agrawal, Solution for a fractional diffusion-wave equation defined in a bounded domain, Fractional order calculus and its applications, Nonlinear Dynam., 29 (2002), 145-155. 1

[3] R. P. Agarwal, V. Lakshmikantham, J. J. Nieto, On the concept of solution for fractional differential equations with uncertainty, Nonlinear Anal., 72 (2010), 2859-2862. 1

[4] M. Z. Ahmad, N. A. Abdul Rahman, Explicit solution of fuzzy differential equations by mean of fuzzy Sumudu transform, Int. J. Appl. Phys. Math., 5 (2015), 86-93. 1, 3.1

[5] M. Z. Ahmad, M. K. Hasan, S. Abbasbandy, Solving fuzzy fractional differential equations using Zadeh's extension principle, Scientific World J., 2013 (2013), 11 pages. 1

[6] A. Ahmadian, M. Suleiman, S. Salahshour, D. Baleanu, A Jacobi operational matrix for solving a fuzzy linear fractional differential equation, Adv. Difference Equ., 2013 (2013), 29 pages. 1

[7] T. Allahviranloo, M. B. Ahmadi, Fuzzy Laplace transforms, Soft Comput., 14 (2010), 235-243. 1

[8] T. Allahviranloo, A. Armand, Z. Gouyandeh, Fuzzy fractional differential equations under generalized fuzzy Caputo derivative, J. Intell. Fuzzy Systems, 26 (2014), 1481-1490. 1

[9] T. Allahviranloo, S. Salahshour, S. Abbasbandy, Explicit solutions of fractional differential equations with uncertainty, Soft Comput., 16 (2012), 297-302. 1

[10] S. Arshad, V. Lupulescu, On the fractional differential equations with uncertainty, Nonlinear Anal., 74 (2011), 36853693. 2.9

[11] B. Bede, S. G. Gal, Almost periodic fuzzy-number-valued functions, Fuzzy Sets and Systems, 147 (2004), 385-403. 5.2

[12] B. Bede, S. G. Gal, Generalizations of the differentiability of fuzzy-number-valued functions with applications to fuzzy differential equations, Fuzzy Sets and Systems, 151 (2005), 581-599. 2.7, 5.2

[13] B. Bede, I. J. Rudas, A. L. Bencsik, First order linear fuzzy differential equations under generalized differentiability, Inform. Sci., 177 (2007), 1648-1662. 2.7

[14] D. A. Benson, S. W. Wheatcraft, M. M. Meerschaert, Application of a fractional advection-dispersion equation, Water Resour. Res., 36 (2000), 1403-1412. 1

[15] A. H. Bhrawy, M. A. Zaky, Shifted fractional-order Jacobi orthogonal functions: application to a system of fractional differential equations, Appl. Math. Model., 40 (2016), 832-845. 1

[16] H. Bulut, H. M. Baskonus, F. B. M. Belgacem, The analytical solution of some fractional ordinary differential equations by the Sumudu transform method, Abstr. Appl. Anal., 2013 (2013), 6 pages. 1

[17] F. Bulut, Ö. Oruç, A. Esen, Numerical Solutions of Fractional System of Partial Differential Equations By Haar Wavelets, Comput. Model. Eng. Sci., 108 (2015), 263-284. 1

[18] V. B. L. Chaurasia, R. S. Dubey, F. B. M. Belgacem, Fractional radial diffusion equation analytical solution via Hankel and Sumudu transforms, Int. J. Math. Eng. Sci. Aero., 3 (2012), 1-10. 1

[19] V. B. L. Chaurasia, J. Singh, Application of Sumudu transform in Schödinger equation occurring in quantum mechanics, Appl. Math. Sci. (Ruse), 4 (2010), 2843-2850. 2.13 
[20] M. Friedman, M. Ma, A. Kandel, Numerical solutions of fuzzy differential and integral equations, Fuzzy modeling and dynamics, Fuzzy Sets and Systems, 106 (1999), 35-48. 2.1

[21] V. Garg, K. Singh, An improved Grunwald-Letnikov fractional differential mask for image texture enhancement, Int. J. Adv. Comput. Sci. Appl., 3 (2012),130-135. 1

[22] W. G. Glöckle, T. F. Nonnenmacher, A fractional calculus approach to self-similar protein dynamics, Biophys. J., 68 (1995), 46-53. 1

[23] A. K. Haydar, Fuzzy Sumudu transform for fuzzy $\mathrm{n}^{\text {th }}$-order derivative and solving fuzzy ordinary differential equations, Int. J. Sci. Res., 4 (2015), 1372-1378. 1

[24] F. Jarad, K. Tas, Application of Sumudu and double Sumudu transforms to Caputo-fractional differential equations, J. Comput. Anal. Appl., 14 (2012), 475-483. 2.13

[25] G. Jumarie, Fractional partial differential equations and modified Riemann-Liouville derivative new methods for solution, J. Appl. Math. Comput., 24 (2007), 31-48. 1

[26] O. Kaleva, A note on fuzzy differential equations, Nonlinear Anal., 64 (2006), 895-900. 2.2

[27] Q. D. Katatbeh, F. B. M. Belgacem, Applications of the Sumudu transform to fractional differential equations, Nonlinear Stud., 18 (2011), 99-112. 1

[28] A. Kaufmann, M. M. Gupta, Introduction to fuzzy arithmetic, With a foreword by Lotfi A. Zadeh, Van Nostrand Reinhold Electrical/Computer Science and Engineering Series, Van Nostrand Reinhold Co., New York, (1985). 2.4

[29] A. Khastan, J. J. Nieto, R. Rodríguez-López, Variation of constant formula for first order fuzzy differential equations, Fuzzy Sets and Systems, 177 (2011), 20-33. 1

[30] A. A. Kilbas, S. A. Marzan, Nonlinear differential equations with the Caputo fractional derivative in the space of continuously differentiable functions, (Russian); translated from Differ. Uravn., 41 (2005), 82-86, Differ. Equ., 41 (2005), 84-89. 1

[31] A. A. Kilbas, H. M. Srivastava, J. J. Trujillo, Theory and applications of fractional differential equations, North-Holland Mathematics Studies, Elsevier Science B.V., Amsterdam, (2006). 1

[32] J. W. Layman, The Hankel transform and some of its properties, J. Integer Seq., 4 (2001), 11 pages. 1

[33] V. Lupulescu, Fractional calculus for interval-valued functions, Fuzzy Sets and Systems, 265 (2015), 63-85. 1

[34] M. Ma, M. Friedman, A. Kandel, Numerical solutions of fuzzy differential equations, Fuzzy Sets and Systems, 105 (1999), 133-138. 2.1

[35] F. Mainardi, The fundamental solutions for the fractional diffusion-wave equation, Appl. Math. Lett., 9 (1996), 23-28. 1

[36] M. Mazandarani, A. V. Kamyad, Modified fractional Euler method for solving fuzzy fractional initial value problem, Commun. Nonlinear Sci. Numer. Simul., 18 (2013), 12-21. 2.2, 2.10, 2.11, 2.12

[37] I. Podlubny, Fractional differential equations, An introduction to fractional derivatives, fractional differential equations, to methods of their solution and some of their applications, Mathematics in Science and Engineering, Academic Press, Inc., San Diego, CA, (1999). 1

[38] S. Salahshour, T. Allahviranloo, S. Abbasbandy, Solving fuzzy fractional differential equations by fuzzy Laplace transforms, Commun. Nonlinear Sci. Numer. Simul., 17 (2012), 1372-1381. 1, 2.8

[39] E. Sousa, C. Li, A weighted finite difference method for the fractional diffusion equation based on the Riemann-Liouville derivative, Appl. Numer. Math., 90 (2015), 22-37. 1

[40] R. A. Spinelli, Numerical inversion of a Laplace transform, SIAM J. Numer. Anal., 3 (1966), 636-649. 1

[41] L. Stefanini, L. Sorini, M. L. Guerra, Parametric representation of fuzzy numbers and application to fuzzy calculus, Fuzzy Sets and Systems, 157 (2006), 2423-2455. 2.1

[42] M. Stynes, J. L. Gracia, A finite difference method for a two-point boundary value problem with a Caputo fractional derivative, IMA J. Numer. Anal., 35 (2015), 698-721. 1

[43] D. Takači, A. Takači, A. Takači, On the operational solutions of fuzzy fractional differential equations, Fract. Calc. Appl. Anal., 17 (2014), 1100-1113. 1

[44] C. J. Tranter, The use of the Mellin transform in finding the stress distribution in an infinite wedge, Quart. J. Mech. Appl. Math. , 1 (1948), 125-130. 1

[45] G. K. Watugala, Sumudu transform: a new integral transform to solve differential equations and control engineering problems, Internat. J. Math. Ed. Sci. Tech., 24 (1993), 35-43. 1

[46] H.-C. Wu, The improper fuzzy Riemann integral and its numerical integration, Inform. Sci., 111 (1998), 109-137. 2.5

[47] J.-P. Xu, Z.-G. Liao, Z.-N. Hu, A class of linear differential dynamical systems with fuzzy initial condition, Fuzzy Sets and Systems, 158 (2007), 2339-2358. 2.1

[48] L. A. Zadeh, Fuzzy sets, Information and Control, 8 (1965), 338-353. 1 